\title{
SMART decisions in fluid management are worth their SALT
}

Gurmeet Singh, MD, MSc, FRCSC, ${ }^{\text {a,b,c,d }}$ and

Andrew Shaw, MB, FRCPC, FRCA, FFICM, FCCM, MMHC ${ }^{\mathrm{c}, \mathrm{d}}$

This Invited Expert Opinion provides a perspective on the following paper: $N$ Engl J Med. 2018;378(9):829-839. https://doi.org/10.1056/NEJMoa1711584.

"In seeking absolute truth, we aim at the unattainable and must be content with broken portions."

\section{— Sir William Osler}

Despite the omnipresence of fluid resuscitation in critical care medicine and cardiothoracic surgery, definitive fluid type recommendations remain elusive. Ideally, fluids should be inexpensive; capable of achieving prolonged, reproducible volume expansion, without extravascular accumulation or producing metabolic derangement; and readily eliminated. "Fluids are drugs,", and each solution's unique properties should guide selection in individual patients. Fluid choice is at the behest of clinician preference, a decision influenced by cost and cognitive bias and encumbered by institutional culture. Emerging evidence challenges, if not confounds, previous physiological-based practice and historic tendencies. New fluid therapy studies addressing distinct populations abound, in efforts to better inform patient-specific management decisions. ${ }^{3-9}$

Equipoise surrounding synthetic colloids, $0.9 \%$ sodium chloride (saline), balanced crystalloids, and albumin use persists. The Isotonic Solutions and Major Adverse Renal Events Trial (SMART) was the first interventional clinical trial demonstrating outcome differences between saline and balanced crystalloid use, particularly major adverse kidney events (MAKEs). ${ }^{10}$ Furthermore, SMART directly correlated acute kidney injury pathogenesis with chloride levels.

A lack of consensus recommendations or practice guidelines reinforces wide variability in volume replacement strategies, and uncertainty endures around fluid choice. In this review, we summarize the recent literature in an attempt to

From the Division of Cardiac Surgery, Departments of ${ }^{\mathrm{a}}$ Critical Care Medicine, ${ }^{\mathrm{b}}$ Surgery, and ${ }^{\mathrm{c}}$ Department of Anesthesiology and Pain Medicine, University of Alberta, and ${ }^{\mathrm{d}}$ Mazankowski Alberta Heart Institute, Edmonton, Alberta, Canada.

Received for publication March 4, 2019; revisions received Dec 24, 2019; accepted for publication Dec 31, 2019; available ahead of print April 9, 2020.

Address for reprints: Andrew Shaw, MB, FRCPC, FRCA, FFICM, FCCM, MMHC, Department of Anesthesiology and Pain Medicine, University of Alberta, 2-150 Clinical Sciences Building, 8440112 St NW, Edmonton, Alberta, Canada T6G 2G3 (E-mail: ashaw2@ualberta.ca).

J Thorac Cardiovasc Surg 2020;160:1250-4 0022-5223/\$36.00

Copyright $(5) 2020$ Published by Elsevier Inc. on behalf of The American Association for Thoracic Surgery

https://doi.org/10.1016/j.jtcvs.2019.12.126

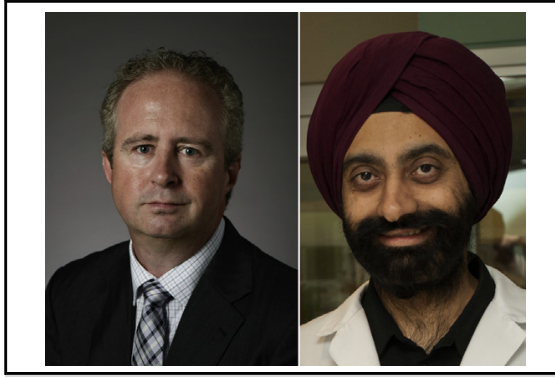

Andrew Shaw, MB, FRCPC, FRCA, FFICM, FCCM, MMHC, and Gurmeet Singh, MD, MSc, FRCSC

CENTRAL MESSAGE

Current evidence favors balanced crystalloid solutions for initial fluid resuscitation in critically ill patients, and cardiothoracic surgical patients would likely benefit from a similar strategy.

This Invited Expert Opinion provides a perspec tive on the following paper: $N$ Engl J Med. 2018;378(9):829-839 NEJMoa1711584.

provide some clarity as to initial solution selection for patients undergoing cardiothoracic surgery.

\section{CRYSTALLOIDS}

Crystalloid management in cardiac surgery lacks uniformity, historically predominated by $0.9 \%$ saline. Studies in unselected critically ill patients suggest an association between acute kidney injury (AKI) and reduced survival with $0.9 \%$ saline. ${ }^{11,12}$ Saline resuscitation results in hyperchloremic metabolic acidosis, ${ }^{13,14}$ and chloride-mediated intrarenal vasoconstriction also may contribute to AKI. ${ }^{15,16}$ Observational data indicate that saline in critical illness is associated with higher rates of $\mathrm{AKI},{ }^{17}$ renal replacement therapy (RRT), ${ }^{17,18}$ and mortality. ${ }^{12,19}$

Evidence of chloride's renal vasoconstrictive effects was demonstrated in a randomized double-blind crossover study 
of healthy adult volunteers who were administered $2 \mathrm{~L}$ of either $0.9 \%$ saline or Plasma-Lyte $148(140 \mathrm{mmol} / \mathrm{L}$ sodium). ${ }^{16}$ Subjects receiving saline experienced sustained hyperchloremia, and magnetic resonance imaging (MRI) found reduced renal artery blood flow velocity and renal cortical perfusion. This study provided a physiological rationale for previous clinical observations.

Based on these data, clinicians have explored balanced crystalloids (including lactated Ringer's and Plasma-Lyte A [Baxter, Missisauga, Ontario, Canada]) as alternatives. Ringer's solution contains $130 \mathrm{mmol} / \mathrm{L}$ sodium, $109 \mathrm{mmol} / \mathrm{L}$ chloride, and lactate as a buffer, with an osmolarity of $272 \mathrm{mOsmol} / \mathrm{L}$ at a $\mathrm{pH}$ of 6.5 . In contrast, PlasmaLyte A contains $140 \mathrm{mmol} / \mathrm{L}$ sodium, $98 \mathrm{mmol} / \mathrm{L}$ chloride, and gluconate and acetate as buffers, with an osmolarity of $294 \mathrm{mOsmol} / \mathrm{L}$ at a pH of 7.4. These more physiologically appealing solutions have recently gained traction in cardiothoracic resuscitation.

A pilot study, the isotonic Solutions Administration Logistical Testing (SALT) trial, an open-label, cluster-randomized multiple-crossover study, compared saline and balanced crystalloids in 974 patients admitted to a single intensive care unit (ICU). ${ }^{20} \mathrm{~A}$ significantly greater incidence of MAKE was observed in the saline group, who received larger-volume resuscitation. ${ }^{20}$ Although mortality and renal injury did not differ between the saline and balanced crystalloid arms, the proposed mechanistic association of hyperchloremia with AKI was correlated with higher serum chloride concentrations, higher peak creatinine, greater incidence of $\mathrm{AKI}$, and more frequent need for RRT in the saline group resuscitated with larger fluid volumes. ${ }^{20}$

Differences in clinical outcomes between the saline and balanced crystalloids arms were finally demonstrated in SMART, a large cluster-randomized, multiple-crossover study. ${ }^{10}$ In this study, 15,802 patients admitted to 5 adult ICUs received either saline or balanced crystalloid (Ringer's lactate or Plasma-Lyte A). The primary outcome was MAKE within 30 days: death, new RRT, or persistent renal dysfunction, defined as a doubling in serum creatinine at 30 days. Secondary clinical outcomes included inhospital mortality before ICU discharge or at 30 , or 60 days; ICU-free days; ventilator-free days; vasopressorfree days; and days alive and free of RRT in the 28-day period after enrollment. Secondary renal outcomes were defined as new RRT, persistent renal dysfunction, stage 2 AKI by Kidney Disease: Improving Global Outcomes (KDIGO) creatinine criteria, peak hospital creatinine, change from baseline to peak creatinine, and creatinine before hospital discharge.

Notably, the incidence of MAKE was significantly lower in the balanced crystalloids group $(14.3 \%$ vs $15.4 \%$; $P=.04)$. The difference in mortality approached but did not achieve statistical significance $(P=.06)$ in favor of balanced crystalloids. New RRT and persistent renal dysfunction did not differ statistically between the 2 groups.

Historically, the hyperchloremia literature has consisted of small, retrospective studies that did not report chloride levels. ${ }^{21}$ These limitations were addressed by SMART. Consistent with pilot study data reported in SALT, SMART data showed fewer patients with hyperchloremia in the balanced crystalloid arm, in keeping with harmful chloride effects, and fewer patients with a serum bicarbonate concentration $<20 \mathrm{mmol} / \mathrm{L}$. The difference between groups was exaggerated in patients receiving larger crystalloid volumes. $^{10}$

Prespecified subgroup analysis showed a greater difference in primary outcomes between study groups in patients receiving larger volumes of fluid resuscitation and in septic patients. In the sepsis subgroup, 30-day in-hospital mortality was significantly reduced in the balanced crystalloid group $(25.2 \%$ vs $29.4 \% ; P=.02){ }^{10}$

One subgroup analysis observation was an apparent point estimate favoring saline in traumatic brain injury and cardiac surgery populations. ${ }^{10}$ The confidence intervals were wide, with crossover to the balanced crystalloid side, making definitive conclusions impossible. One potential explanation may be differing indications for RRT in cardiac surgery patients; in these patients, RRT is likely to be instituted earlier for volume overload, as opposed to metabolic (nitrogenous waste clearance) reasons.

SMART was the first large interventional trial to demonstrate an important outcome difference based on crystalloid choice, bolstered by a large sample size powered to elicit such distinctions. The study is consistent with physiological and mechanistic considerations of saline's harmful effects. Furthermore, an economic analysis of calcium-free balanced crystalloid solution (Plasma-Lyte) adoption reduced complication-related costs in patients with systemic inflammatory response syndrome (SIRS). ${ }^{22}$ Based on the current evidence, it appears advisable to favor balanced crystalloids over saline for subgroups of cardiothoracic surgery patients, especially those with large volume requirements and those at risk for AKI. Specific confirmation in large numbers of cardiac surgery patients would further cement this recommendation, but whether cardiac surgeons and intensivists would be comfortable randomizing patients to a group receiving only $0.9 \%$ saline is unclear.

\section{SYNTHETIC COLLOIDS}

Cardiopulmonary bypass stimulates profound SIRS. Regardless of fluid choice, edema is a consequence, especially under coexist inflammatory conditions, such as systemic infection (eg, endocarditis). ${ }^{1}$ The early or contact activation phase of inflammation is purportedly a 
consequence of contact of blood components with nonbiological synthetic surfaces. The contact system of primary plasma proteins Factor XII (Hageman factor), Factor XI, prekallikrein, and high molecular weight kinongen; intrinsic and extrinsic coagulation systems; complement; and fibrinolysis are all invoked. Cellular components, such as endothelial cells, leukocytes, neutrophils, monocytes, lymphocytes, and platelets, are also activated by extracorporeal perfusion. The later inflammatory phase is felt to be mediated by ischemia-reperfusion and endotoxins. ${ }^{23}$ The inflammatory assault is extensive, potentially adversely affecting all organ systems. Various pharmacologic and technical strategies have been investigated to attenuate induced SIRS. ${ }^{24}$

Synthetic colloids are conceptually attractive in a proinflammatory milieu, with potentially less absolute volume requirements and the additional benefit of remaining within the intravascular space. Hydroxyethyl starch (HES) solutions have been used for decades for volume expansion, more commonly in Europe and Asia than in North America. HES is derived from potato or waxy maize, with hydroxyl groups on the amylopectin molecule substituted by hydroxyethyl residues. ${ }^{2}$ HES solutions are designated with 3 numbers: the first digit represents solution concentration (eg, 6\%), the second number denotes mean molecular weight (eg, 130), and the final number is the degree of molar substitution (eg, 0.4), the number of hydroxyethyl residues per unit glucose.

Early HES versions contained a large, highly-substituted molecule, resulting in long plasma half-life, consequentially impairing coagulation and predisposing to $\mathrm{AKI} .{ }^{25}$ Newer HES solutions, referred to as third-generation, have significantly lower molecular weight and molar substitution. ${ }^{25}$ A lower molar substitution molecule is more readily hydrolyzed, reducing the half-life to approximately 12 hours. $^{2}$

In 2011, several published papers on HES were retracted, with extensive media coverage. ${ }^{26}$ A particular focus on research misconduct attributed to Joachim Boldt resulted in dozens of publication retractions. ${ }^{27}$ Around the same time, the Scandinavian Starch for Severe Sepsis/Septic Shock (6S) trial reported increased 90-day mortality and increased likelihood of RRT in patients with severe sepsis resuscitated with HES. ${ }^{28}$ Conversely, the Crystalloid versus Hydroxyethyl Starch Trial (CHEST) did not find a difference in 90-day mortality between HES and saline in critically ill patients. ${ }^{29}$ Consistent with 6 S, CHEST revealed greater RRT in the HES arm. Following a public workshop and data review, the US Food and Drug Administration (FDA) issued a black-box warning for increased mortality risk and RRT with HES solutions, particularly for septic patients. (https://wayback.archive-it.org/7993/2017011 2095648/http://www.fda.gov/BiologicsBloodVaccines/ SafetyAvailability/ucm 358271.htm). The FDA also included an additional warning for excessive bleeding in cardiac surgery patients undergoing cardiopulmonary bypass.

Contrarily, the Colloids Versus Crystalloids for the Resuscitation of the Critically Ill (CRISTAL) trial reported lower 90-day mortality in the colloid arm. ${ }^{30}$ Furthermore, the most recent Cochrane review evaluating colloids versus crystalloids for fluid resuscitation of critically ill individuals found little or no difference in all-cause mortality based on moderate-certainty evidence. ${ }^{31}$ The authors noted that this contradicted the previous Cochrane review that reported possible increased mortality with HES. Perhaps a reevaluation of HES in cardiac surgery is merited.

As highlighted by the FDA, increased blood loss has been linked to HES use (https://wayback.archive-it.org/7993/ 20170112095648/http://www.fda.gov/BiologicsBlood Vaccines/Safety Availability/ucm358271.htm). This assessment was based on a meta-analysis with insufficient available data comparing multiple preparations of HES, with only 4 studies using the newer $6 \% / 130 / 0.4$ solution. ${ }^{32}$

Proponents of HES argue that third-generation starches are safe in cardiac surgery, ${ }^{25}$ as coagulation effects are similar to those of albumin and crystalloids, and studies have refuted increased hemorrhage or transfusion requirements linked to older starch products. ${ }^{33,34}$ HES critics point out that no data describe improved outcomes, and fairly convincing data report hazards in patients in similar clinical circumstances.

A single-center retrospective study of 606 patients found double the risk of AKI in HES versus crystalloid groups $\left(21.5 \%\right.$ vs $9.5 \%$, respectively). ${ }^{35}$ Another singleinstitution prospective observational study of 6478 cardiac surgery patients, sequentially analyzed over 3 time periods, reported significantly more RRT with HES compared with crystalloids. ${ }^{36}$

Conversely, a small single-center retrospective study analyzed renal injury biomarkers and found no suggestion of HES-mediated tubular injury, although there was a nonsignificant tendency toward greater blood loss and higher vasopressor dosing. ${ }^{37}$ A large prospective observational study of registry data collected across 3 institutions with a total of 17,742 patients over 7 years failed to show increased RRT or a negative impact on 30-day mortality with HES use. ${ }^{38}$ Interestingly, these authors found worse outcomes with albumin. A further multicenter (23 sites) prospective observational cohort study including 1058 patients, 350 of whom received HES, did not have an increased risk of AKI or RRT after multivariable risk adjustment and propensity score matching. ${ }^{39}$

Currently, starch safety has not been definitively established for cardiac surgery patients. Biological plausibility and some studies suggest a potential role; however, wellconducted trials are lacking. Until conclusive evidence of HES benefit over crystalloids emerges, given the substantial cost difference and evidence of harm in other, similar 
patient populations, we believe that HES should be confined to clinical trial populations in cardiac surgical practice.

\section{ALBUMIN}

Albumin regulates plasma oncotic pressure and compartmental fluid distribution. ${ }^{40}$ The cost and perceived risks of human albumin solutions deter wider use as an adjunct colloid. Potential prion transmission and possible increased AKI risk in patients with preexisting renal dysfunction are other considerations. ${ }^{41}$ The SAFE study, which randomized nearly 7000 critically ill patients to receive either $4 \%$ albumin or $0.9 \%$ saline, demonstrated neither a survival advantage nor a difference in RRT application. ${ }^{42}$

Theoretical benefits of albumin in cardiac surgery include inflammation and platelet activation amelioration. ${ }^{43}$ Purported advantages include antioxidant action as a consequence of nitric oxide scavenging ${ }^{41}$ and positive effects on the endothelial glycocalyx degradation pathway. ${ }^{43}$ The concept of albumin as a drug rather than a fluid solution justifies closer examination.

Greater risk of RRT with albumin usage in cardiac surgery has been reported, but this study was heterogenous, as patients received both $20 \%$ and/or $5 \%$ albumin. ${ }^{38} \mathrm{~Pa}-$ tients receiving $20 \%$ albumin had a significant increase in RRT requirements, compared with those believed to have received $5 \%$ albumin. No propensity matching was performed to compare crystalloids with 5\% albumin, leading to a high possibility of uncontrolled confounding.

A recent observational study compared a cohort of 1095 cardiac surgery patients who received 5\% albumin in addition to crystalloids with an equivalent number of patients who received only crystalloid, using propensity score matching and found significantly lower in-hospital mortality and all-cause 30-day readmissions for the albumin group, with no association with $\mathrm{AKI} .{ }^{43}$ The albumin arm had significantly fewer large hemoglobin drops, although no difference in transfusion rates. As with other studies, there was no firm conclusion that improved outcomes were associated with any meaningful reduction in volume administration. ${ }^{43}$

\section{CONCLUSIONS}

Evidence is still accumulating for ideal fluid replenishment strategies and solutions in cardiac surgery. Observational and clinical data confirm adverse renal vasoactive effects of hyperchloremia, with associated worse outcomes. In SMART, the effects were further exaggerated in favor of balanced crystalloids over saline when larger volumes were administered. ${ }^{10}$ Therefore, current data favor balanced crystalloid solutions over saline.

The theoretical benefit of additional colloids to attenuate crystalloid hypervolemia requires further investigation. It is possible, but not probable, that the harmful effects of newer HES solutions might have been overestimated in cardiac surgery, but a current paucity of evidence prevents firm recommendations. The undoubted hazard in other patients experiencing significant inflammatory physiology is undeniable, and thus clinicians may choose to avoid these products until there is clear evidence of a favorable risk-benefit ratio.

Recent evidence appears to contradict earlier reports of albumin associated renal hazard. It is difficult to reconcile toxicity of the most abundant circulating human protein, but once again, more robust data are needed before recommending a specific tactic using albumin as a colloid supplement.

In conclusion, adult cardiac surgery patients' IV fluid needs are likely best served by a balanced crystalloid solution, supplemented with albumin when large volumes are administered. We fully accept that this recommendation is largely opinion, rather than conclusively supported by evidence. Nevertheless, clinicians must make decisions, and we offer this perspective in an effort to help those who, like us, are privileged to serve cardiac surgical patients daily.

\section{Conflict of Interest Statement}

Authors have nothing to disclose with regard to commercial support.

\section{References}

1. Myburgh JA, Mythen MG. Resuscitation fluids. N Engl J Med. 2013;369: 1243-51.

2. Raghunathan K, Miller TE, Shaw AD. Intravenous starches: is suspension the best solution? Anesth Analg. 2014;119:731-6.

3. Wu MC, Liao TY, Lee EM, Chen YS, Hsu WT, Lee MG, et al. Administration of hypertonic solutions for hemorrhagic shock: a systematic review and metaanalysis of clinical trials. Anesth Analg. 2017;125:1549-57.

4. Sethi M, Owyang CG, Meyers C, Parekh R, Shah KH, Manini AF. Choice of resuscitative fluids and mortality in emergency department patients with sepsis. Am J Emerg Med. 2018;36:625-9.

5. Pfortmueller CA, Schefold JC. Hypertonic saline in critical illness-a systematic review. J Crit Care. 2017;42:168-77.

6. Thompson M, McIntyre L, Hutton B, Tran A, Wolfe D, Hutchison J, et al. Comparison of crystalloid resuscitation fluids for treatment of acute brain injury: a clinical and pre-clinical systematic review and network meta-analysis protocol. Syst Rev. 2018;7:125.

7. Winters ME, Sherwin R, Vilke GM, Wardi G. What is the preferred resuscitation fluid for patients with severe sepsis and septic shock? J Emerg Med. 2017;53: 928-39.

8. Meyhoff TS, Møller MH, Hjortrup PB, Cronhjort M, Perner A, Wetterslev J. Lower vs. higher fluid volumes in sepsis-protocol for a systematic review with meta-analysis. Acta Anaesthesiol Scand. 2017;61:942-51.

9. Eljaiek R, Heylbroeck C, Dubois MJ. Albumin administration for fluid resuscitation in burn patients: a systematic review and meta-analysis. Burns. 2017;43: 17-24.

10. Semler MW, Self WH, Wanderer JP, Ehrenfeld JM, Wang L, Byrne DW, et al. Balanced crystalloids versus saline in critically ill adults. N Engl J Med. 2018; 378:829-39.

11. Boniatti MM, Cardoso PRC, Castilho RK, Vieira SR. Is hyperchloremia associated with mortality in critically ill patients? A prospective cohort study. J Crit Care. 2011;26:175-9.

12. Shaw AD, Raghunathan K, Peyerl FW, Munson SH, Paluszkiewicz SM Schermer CR. Association between intravenous chloride load during resuscitation and in-hospital mortality among patients with SIRS. Intensive Care Med. 2014;40:1897-905. 
13. Scheingraber S, Rehm M, Sehmisch C, Finsterer U. Rapid saline infusion produces hyperchloremic acidosis in patients undergoing gynecologic surgery. Anesthesiology. 1999;90:1265-70.

14. Waters JH, Gottlieb A, Schoenwald P, Popovich MJ, Sprung J, Nelson DR Normal saline versus lactated Ringer's solution for intraoperative fluid management in patients undergoing abdominal aortic aneurysm repair: an outcome study. Anesth Analg. 2001;93:817-22.

15. Wilcox CS. Regulation of renal blood flow by plasma chloride. J Clin Invest. 1983;71:726-35.

16. Chowdhury AH, Cox EF, Francis ST, Lobo DN. A randomized, controlled, double-blind crossover study on the effects of 2 - $\mathrm{L}$ infusions of $0.9 \%$ saline and plasma-lyte 148 on renal blood flow velocity and renal cortical tissue perfusion in healthy volunteers. Ann Surg. 2012;256:18-24.

17. Yunos NM, Bellomo R, Hegarty C, Story D, Ho L, Bailey M. Association between a chloride-liberal vs chloride-restrictive intravenous fluid administration strategy and kidney injury in critically ill adults. JAMA. 2012;308:1566-72.

18. Krajewski ML, Raghunathan K, Paluszkiewicz SM, Schermer CR, Shaw AD Meta-analysis of high- versus low-chloride content in perioperative and critical care fluid resuscitation. Br J Surg. 2015;102:24-36.

19. Raghunathan K, Shaw A, Nathanson B, Stürmer T, Brookhart A, Stefan MS, et al. Association between the choice of IV crystalloid and in-hospital mortality among critically ill adults with sepsis. Crit Care Med. 2014;42:1585-91.

20. Semler MW, Wanderer JP, Ehrenfeld JM, Stollings JL, Self WH, Siew ED, et al. Balanced crystalloids versus saline in the intensive care unit. The SALT randomized trial. Am J Respir Crit Care Med. 2017;195:1362-72.

21. Bandak G, Kashani KB. Chloride in intensive care units: a key electrolyte F1000Res. 2017;6:1930.

22. Laplante S, Makhija DU, Munson SH, Khangulov VS, Peyerl FW, Paluszkiewicz SM, et al. Impact of fluid choice in systemic inflammatory response syndrome patients on hospital cost savings. Pharmacoecon Open. 2018;2:325-35

23. Warren OJ, Smith AJ, Alexiou C, Rogers PL, Jawad N, Vincent C, et al. The inflammatory response to cardiopulmonary bypass, part 1: mechanisms of pathogenesis. J Cardiothorac Vasc Anesth. 2009;23:223-31.

24. Warren OJ, Watret AL, de Wit KL, Alexiou C, Vincent C, Darzi AW, et al. The inflammatory response to cardiopulmonary bypass, part 2: anti-inflammatory therapeutic strategies. J Cardiothorac Vasc Anesth. 2009;23:384-93.

25. McConnell M, Baisden J, Duncan AE. Pro: third-generation hydroxyethyl starch solution is safe and effective for plasma volume expansion during cardiac surgery. J Cardiothorac Vasc Anesth. 2018;32:570-5.

26. Shafer SL. Shadow of doubt. Anesth Analg. 2011;112:498-500.

27. McHugh UM, Yentis SM. An analysis of retractions of papers authored by Scott Reuben, Joachim Boldt and Yoshitaka Fujii. Anaesthesia. 2019:74:17-21.

28. Perner A, Haase N, Guttormsen AB, Tenhunen J, Klemenzson G, Ảneman A et al. Hydroxyethyl starch 130/0.42 versus Ringer's acetate in severe sepsis. N Engl J Med. 2012;367:124-34.
29. Myburgh JA, Finfer S, Bellomo R, Billot L, Cass A, Gattas D, et al. Hydroxyethyl starch or saline for fluid resuscitation in intensive care. N Engl J Med. 2012;367: 1901-11.

30. Annane D, Siami S, Jaber S, Martin C, Elatrous S, Declère AD. Effects of fluid resuscitation with colloids vs crystalloids on mortality in critically ill patients presenting with hypovolemic shock: the CRISTAL randomized trial. JAMA. 2013;310:1809-17.

31. Lewis SR, Pritchard MW, Evans DJ, Butler AR, Alderson P, Smith AF, et al. Colloids versus crystalloids for fluid resuscitation in critically ill people. Cochrane Database Syst Rev. 2018;8:CD000567.

32. Navickis RJ, Haynes GR, Wilkes MM. Effect of hydroxyethyl starch on bleeding after cardiopulmonary bypass: a meta-analysis of randomized trials. J Thorac Cardiovasc Surg. 2012;144:223-30.

33. Jacob M, Fellahi JL, Chappell D, Kurz A. The impact of hydroxyethyl starches in cardiac surgery: a meta-analysis. Crit Care. 2014;18:656.

34. Van Der Linden P, James M, Mythen M, Weiskopf RB. Safety of modern starches used during surgery. Anesth Analg. 2013;116:35-48.

35. Lagny MG, Roediger L, Koch JN, Dubois F, Senard M, Donneau AF, et al. Hydroxyethyl starch 130/0.4 and the risk of acute kidney injury after cardiopulmonary bypass: a single-center retrospective study. J Cardiothorac Vasc Anesth. 2016;30:869-75.

36. Bayer O, Schwarzkopf D, Doenst T, Cook D, Kabisch B, Schelenz C, et al. Peri operative fluid therapy with tetrastarch and gelatin in cardiac surgery-a prospective sequential analysis. Crit Care Med. 2013;41:2532-42.

37. Datzmann T, Hoenicka M, Reinelt H, Liebold A, Gorki H. Influence of $6 \%$ hydroxyethyl starch 130/0.4 versus crystalloid solution on structural renal damage markers after coronary artery bypass grafting: a post hoc subgroup analysis of a prospective trial. J Cardiothorac Vasc Anesth. 2018;32:205-11.

38. Ryhammer PK, Tang M, Hoffmann-Petersen J, Leonaviciute D, Greisen J, Storebjerg Gissel M, et al. Colloids in cardiac surgery-friend or foe? J Cardiothorac Vasc Anesth. 2017:31:1639-48.

39. Vives M, Callejas R, Duque P, Echarri G, Wijeysundera DN, Hernandez A, et al Modern hydroxyethyl starch and acute kidney injury after cardiac surgery: a prospective multicentre cohort. Br J Anaesth. 2016;117:458-63.

40. Fanali G, di Masi A, Trezza V, Marino M, Fasano M, Ascenzi P. Human serum albumin: from bench to bedside. Mol Aspects Med. 2012;33:209-90.

41. Jiang Y, Shaw AD. Albumin supplementation as a therapeutic strategy in cardiac surgery: useful tool or expensive hobby? Anesthesiology. 2016;124: 983-5.

42. Finfer S, Bellomo R, Boyce N, French J, Myburgh J, Norton R. A comparison of albumin and saline for fluid resuscitation in the intensive care unit. $N$ Engl J Med. 2004:350:2247-56.

43. Kingeter AJ, Raghunathan K, Munson SH, Hayashida DK, Zhang X, Iyengar S, et al. Association between albumin administration and survival in cardiac surgery: a retrospective cohort study. Can J Anesth. 2018;65:1218-27. 